\title{
NIDCAP: A Psychoneuroendocrine Perspective
}

\section{Goldstein Ferber S', McAnulty $\mathbf{G}^{2}$, Klinge $\mathbf{G}^{3}$, Weller $\mathrm{A}^{1}$,} Als $\mathrm{H}^{2}$

\author{
Department of Psychology and Gonda Brain Research Center, Bar llan \\ University, Israel. \\ 2 Department of Psychiatry, Boston Children's Hospital and Harvard Medical \\ School, USA \\ ${ }^{3}$ Department of Neonatology, Schneider Children's Medical Center, Sackler \\ Medical School, Tel Aviv University, Israel.
}

\section{Aims}

This presentation outlines the pathways through which NIDCAP promotes hypothalamic control of cognitive development by attenuating inevitable NICU stress, thus regulating over-arousal, flaccidity, and shutdown.

\section{Methods}

By integrating past decade's research on the hypothalamus and preterm infants.

\section{Results}

HPA - Three hormones regulated by the HPA, Melatonin, Cortisol and Oxytocin, are implicated in the preterm infant's NICU experience: Melatonin as regulator of circadian rhythm maturation and in turn influencer of cognitive development ${ }^{1}$ (Bayley Scales); Cortisol as regulator of stress modulation, and Oxytocin as mediator of parent infant affectionate relationship and bonding. Moreover, preterm newborns lack the capacity to generate a full adrenocortical response to stress or illness, which conversely, later in development turns into a disproportionate increase in glucocorticoid action and over-arousal states.

HPT - Congenital hypothyroidism is frequently associated with the development of intellectual disability. Often it is involved in the association of lower gestational age at birth and impaired cognitive and self-regulation functioning later on. ${ }^{2}$ In addition, inadequate HPA stress regulation in preterm infants further compromises their HPT function through HPA-HPT cross-connectivity.

HPG - Fluctuations of progesterone and estrogen levels are relevant for females' optimal HPA reactivity. In males, early programing of testosterone levels affects HPA and testosterone reprogramming during puberty and is necessary for adaptive HPA reactivity in adulthood. ${ }^{3}$

Thus, all three axes of the hypothalamus and their 'crosstalk' are relevant, either by direct or by secondary impact, ${ }^{3}$ to the understanding of prematurity and its resulting compromised cognitive and self-regulation development. The variability documented in the methylation of the genes of these metabolic neurochemicals' receptors, OXTR, NR3C1 and Nrf2, may suggest that hypothalamic regulation works in concert with the epigenetic effects to bring about an optimal range of receptor availability if supported by optimal environmental inputs. Reduction of stress may bring about compensatory transcription processes in the hypothalamic pathways. Thus, ameliorative alterations of the epigenome are possible with balanced environmental inputs. ${ }^{4,5}$

\section{Relevance to NIDCAP}

By affecting stress reactivity and the HPA, NIDCAP methods appear to affect the function of the hypothalamic axes, which show association and causality pathways with cognitive and self-regulation functions. It is postulated that feedback mechanisms and correction signals of hypothalamus-based homeostatic modulating functions account for the stress reduction brought about by very early NIDCAP intervention.

\section{Conclusion}

The balancing effects of NIDCAP are fed back to the hypothalamus for correction of the signaling to prefrontal cortex, the seat of executive functions. Given their bi-directional pathways, in turn the neuro-hormone and neuropeptide production levels adjust accordingly.

References

1. Ferber SG, Als H, McAnulty G, Peretz H, Zisapel N. Melatonin and mental capacities in newborn infants. Journal of Pediatrics. 2011, 159(1):99-103.e1. DOI. 10.1016/j. jpeds.2010.12.032

2. McBryde M, Fitzallen GC, Liley HG, Taylor HG, Bora S. Academic outcomes of schoolsged children born preterm: A systematic review and meta-analysis. JAMA Network Open. 2020, 3(4):e202027. DOI: 10.1001/jamanetworkopen.2020.2027.

3. Oyola MG, Handa RJ. Hypothalamic-pituitary-adrenal and hypothalamic-pituitary-gonadal axes: sex differences in regulation of stress responsivity. Stress. 2017 Sep;20(5):476494. DOI: $10.1080 / 10253890.2017 .1369523$

4. Goldstein Ferber S, Trezza V, Weller A. Early life stress and development of the endocannabinoid system: A bidirectional process in programming future coping. Dev Psychobiol. 2021, 63(2):143-52. DOI:10.1002/dev.21944

5. Ferber SG, Roth TL, Weller A. Epigenetic fragility of the endocannabinoid system under stress: risk for mood disorders and pharmacogenomic implications. Epigenomics. 2020, 14(8):657-660. doi.org/10.2217/epi-2020-0037

\section{NIDCAP Trainers Meeting Feedback}

"We would like to see more clinical practice sessions, open discussion around APIB and small discussion groups." 